\title{
Percutaneous embolization of congenital portosystemic venous shunt in an infant with respiratory distress
}

\author{
Fatoş Alkan¹, Fatih Düzgün², Hasan Yüksel ${ }^{3}$, Serdar Tarhan², Şenol Coşkun ${ }^{1}$ \\ ${ }^{1}$ Division of Pediatric Cardiology, ${ }^{3}$ Division of Pediatric Allergy and Pulmonology Department of Pediatrics, ${ }^{2}$ Department \\ of Radiology, Celal Bayar University, School of Medicine, Manisa, Turkey. E-mail: fatos.alkan@hotmail.com \\ Received: 19th July 2017, Accepted: 22nd August 2017
}

SUMMARY: Alkan F, Düzgün F, Yüksel H, Tarhan S, Coşkun Ş. Percutaneous embolization of congenital portosystemic venous shunt in an infant with respiratory distress. Turk J Pediatr 2018; 60: 456-459.

A 5-month-old boy with progressive respiratory distress was admitted to our hospital. Physical examination revealed mild tachypnea and retraction. The left main bronchus was found as severely collapsed between the right pulmonary artery and the descending aorta, on the bronchoscopic evaluation. Further evaluation revealed persistent ductus venosus (PDV). As in the fetal period the ductus venosus arises from the posterior aspect of the left portal vein, a PDV is considered another type of intrahepatic portosystemic shunt. Therefore, he was diagnosed with congenital portosystemic venous shunt, leading to persistent respiratory distress. The PDV was closed with Amplatzer vascular plug II, and then he had immediate clinical improvement. Congenital portosystemic venous shunts (CPSS) are rare vascular malformations associated with severe complications. Here we presented a case with progressive respiratory distress as a result of CPSS and rapid improvement after embolization.

Key words: portosystemic venous shunt, persistent ductus venosus, amplatzer vascular plug II.

Congenital portosystemic shunts (CPSS) are rare anomalies. These defects have been classified into extrahepatic and intrahepatic based on the location of the shunts. Persistent ductus venosus or abnormal intrahepatic connections are considered as the major leading factors of intrahepatic shunts. ${ }^{1}$ Ultrasonography (US), Computed Tomography (CT) and Magnetic Resonance (MR) angiography are the tools used for diagnosis. ${ }^{2}$ Complications of CPSS may manifest in early infancy or later in life, including neonatal hyperammonemia, cholestasis, hypergalactosemia, liver tumors (benign or malignant), portosystemic encephalopathy, right heart failure, pulmonary arterial hypertension and hepatopulmonary syndrome. ${ }^{3}$ In this report we presented an infant with progressive respiratory distress due to complication of CPSS leading to persistent ductus venosus (PDV) successfully treated by a transvenous amplatzer vascular plug II embolization.

\section{Case Report}

A 5-month-old boy with progressive respiratory distress was admitted to our hospital. He had severe cough, increased work of breathing, accompanied by decreased oral intake. Physical examination revealed tachypnea (60-70 breaths/ min), retraction, and oxygen saturation of $98 \%$ on room air and a pulse rate of $145-160 \mathrm{bpm}$. He had hepatomegaly, without splenomegaly. A chest graph demonstrated an enlarged cardiac silhouette and increased pulmonary vascular markings indicating increased lung circulation. Echocardiography demonstrated normal ventricular function but increased in size. No additional cardiac defect was found. However, on subcostal view an extra cardiac vascular structure opening to the right atrium (RA) on the posterior lateral side of the inferior vena cave was observed. On bronchoscopic exam, it was found that the left main bronchus was severely compressed between the right 


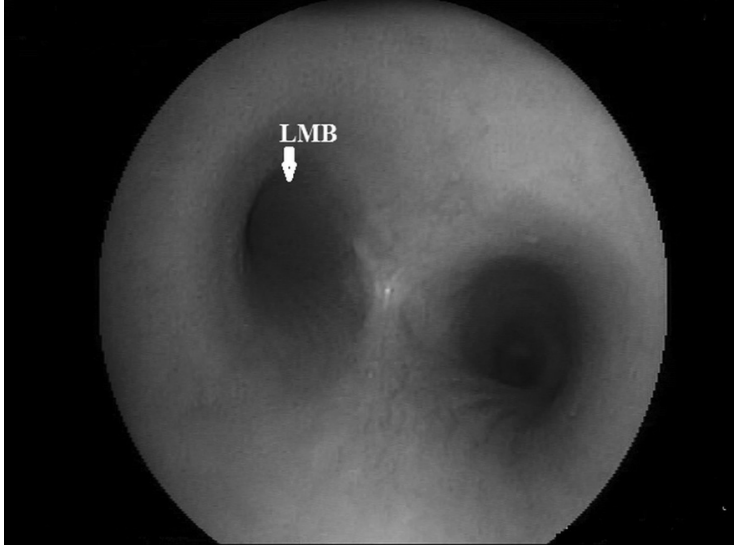

Fig. 1. Collapsing of the left main bronchi (LMB) on the bronchoscopic exam.

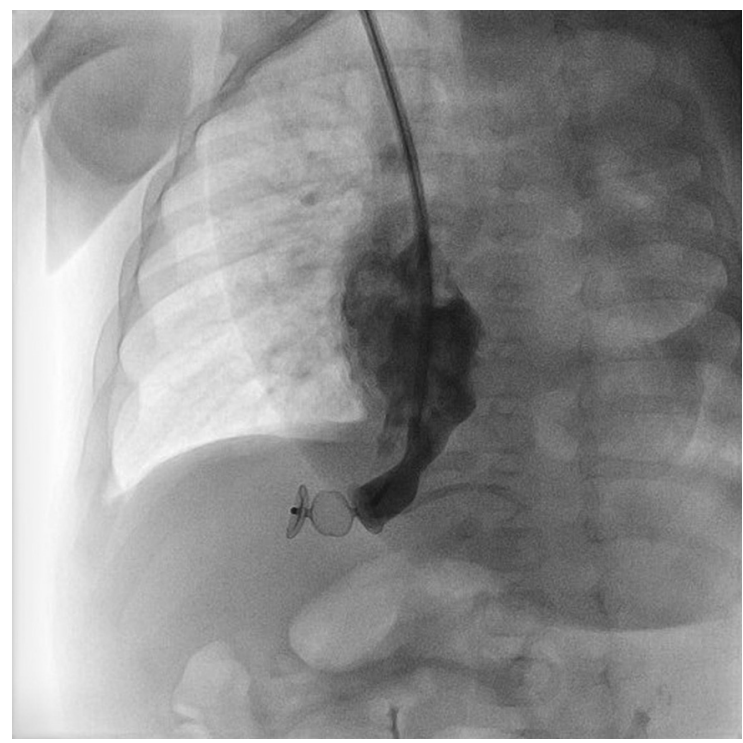

Fig. 2. Persistent ductus venosus with an aneurysmatic structure from left portal vein on the sagittal MIP section of contrasted CT.

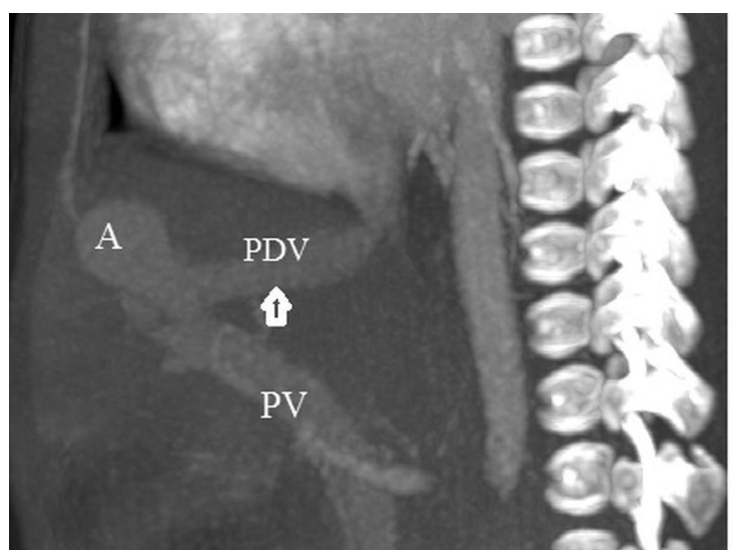

Fig. 3. Abnormal biphasic flow of portal vein on the Doppler Ultrasonogram.

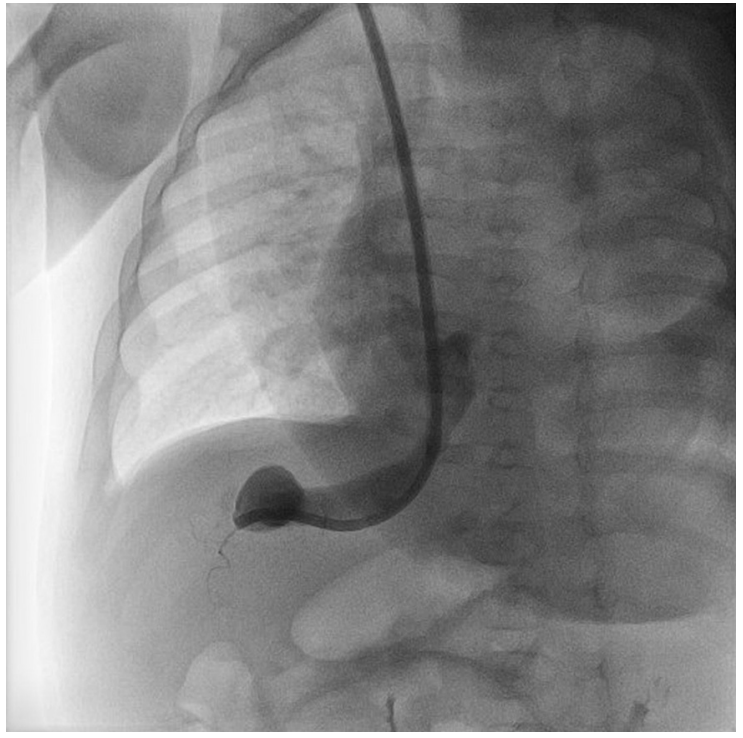

Fig. 4. Device embolization, anterior-posterior view.

A) Retrograde introduction of the implantation catheter through the inferior caval vein to the ductus venosus.

B) The device was deployed into the lumen of the ductus venosus and after detachment revealed no residual shunt.

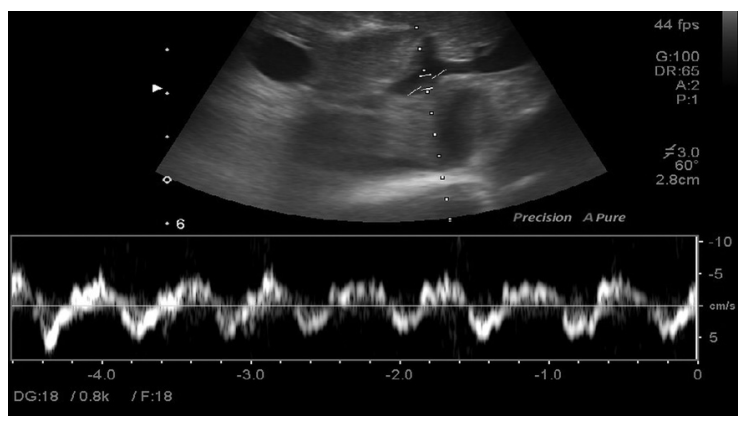

pulmonary artery and descending aorta (Fig. 1). CT angiography confirmed the collapsed left main bronchus due to dilated aorta and PDV, which connected from the left portal vein to the inferior vena cava (IVC) (Fig. 2). Doppler ultrasonogram showed a PDV with $12 \times 6 \times 5 \mathrm{~mm}$ in diameters, opening into the IVC and no evidence of portal hypertension (Fig. 3 ). Right heart catheterization confirmed the diagnosis of the large PDV, which connected from the left portal vein to the IVC (Fig. $4 \mathrm{~A}$ ). The diagnosis of CPSS was certain by the finding of PDV. The operative risk was estimated as high; therefore, embolization by an Amplatzer vascular plug type device II was carefully planned. The PDV was entered from the internal jugular vein access. Test balloon occlusion showed adequate hepatic 
portal circulation and no increase in portal pressure from the basal $10 \mathrm{mmHg}$. Through a 5F sheath, a $10 \mathrm{~mm}$ Amplatzer vascular plug II was used (Fig. 4B). Repeated angiography confirmed complete occlusion of the PDV (Fig. 4B). There were no complications. He was given only acetylsalicylic acid. On the second day the patient was discharged. A subsequent abdominal US demonstrated resolution of the PDV. After the procedure, the infant started to improve clinically and he is stable for 5 months.

Written informed consent was obtained from patients parents.

\section{Discussion}

Congenital portosystemic shunts are rare malformations and are classified mainly as: extrahepatic (abernethy malformation) or intrahepatic (PDV).1,4 These defects are abnormal intrahepatic vascular formations between the portal vein and hepatic veins or inferior vena cava. There are 4 types reported in the literature. ${ }^{5}$ Type 1 is a single large vessel connection between right portal vein and IVC, type 2 is a local peripheral shunt consisting of branches of the portal vein and the hepatic veins, type 3 is an aneurismal structure between the peripheral portal vein and the hepatic veins and type 4 is defined as multiple vessels between the portal vein and the hepatic veins distributed in both lobes $^{5}$ Although rare, a PDV can function like a intrahepatic portosystemic shunt. In the fetus, the ductus venosus arises from left portal vein, opposite the opening of the umbilical vein, and drains into a hepatic vein. In most full terms, it closes spontaneously in the post-natal period. ${ }^{6}$ If there are any congenital heart defects present or if the baby is preterm, the closure time may delay. ${ }^{1}$ Thus it can result into a portosystemic shunt. Congenital intrahepatic forms are rarer than the acquired ones. ${ }^{7}$ Due to improved diagnostic imaging techniques, incidence has increased recently. ${ }^{7}$ CPSS is diagnosed either incidentally on abdominal US or diagnosed after congestive heart failure occurs due to significant intra-hepatic short circuiting of blood. ${ }^{3}$ In other cases, they may cause metabolic disorders and various organ and system failures, such as liver, central nervous system, or respiratory tract. $^{3}$ Our patient suffered from respiratory distress due to left pulmonary bronchial pressure of the dilated aorta. Due to the wide variability in clinical presentation, imaging plays a crucial role to recognize the shunt and related malformations and also during follow-up of patients with CPSS. ${ }^{2}$ US especially US with color Doppler is the initial preferential method. MR angiography could provide additional information about the hepatic vascular and parenchymal abnormalities. Although using methods that minimize exposure to ionizing radiation is preferable in pediatric patients, this imaging alone is insufficient. CT angiography is considered the first choice examination because this method displays even small vascular branches compatible with a portocaval shunt. ${ }^{2}$ CT also allows imaging of intrahepatic lesions of very small dimensions, as we presented in our case.

Usually spontaneous closure of portosystemic shunts can occur within the first 2 years of age. ${ }^{1}$ Asymptomatic ones may remain until childhood. Invasive treatment with shunt closure in patients with CPSS is recommended with regard to the severity of complications and its potential reversibility after shunt closure. ${ }^{8}$ On the other hand, if there is severe pulmonary hypertension or right heart failure, treatment either by transcatheter or surgical approach should be considered. ${ }^{8}$. Our patient was at risk for high output cardiac failure due to increased venous return caused by the low resistance hepatic circulation. Percutaneous management was less invasive than an open surgical approach. Therefore, we preferred this treatment in our patient. It should be checked that there is enough portal vein hepatic ramification and absence of portal hypertension should be assured before procedure.

In conclusion, CPSS can lead to progressive respiratory distress due to increased cardiac output. The transcatheter closing of CPSS with Amplatzer vascular plug II is a simple, possible and confident technique. The percutaneous closure of CPSS with an Amplatzer vascular plug type device II can be considered as a therapeutic option in cases with favorable anatomy. 


\section{REFERENCES}

1. Stringer MD. The clinical anatomy of congenital portosystemic venous shunts. Clin Anat 2008; 21: 147-157.

2. Alonso-Gamarra E, Parr'on M, $\mathrm{P}^{\prime}$ erez A, Prieto C, Hierro L, López-Santamaría M. Clinical and radiologic manifestations of congenital extrahepatic portosystemic shunts: a comprehensive review. Radiographics 2011; 31: $707-722$

3. Franchi-Abella S, Branchereau S, Lambert V, et al. Complications of congenital portosystemic shunts in children: therapeutic options and outcomes. J Pediatr Gastroenterol Nutr 2010; 51: 322-330.

4. Yoshimoto Y, Shimizu R, Saeki T, et al. Patent ductus venosus in children: A case report and review of the literature. J Pediatr Surg 2004; 39: E1-E5.
5. Park JH, Cha SH, Han JK, Han MC. Intrahepatic portosystemic venous shunt. AJR Am J Roentgenol 1990; 155: 527-528.

6. Meyer WW, Lind J. The ductus venosus and the mechanism of its closure. Arch Dis Child 1966; 41: 597-605.

7. Han BH, Park SB, Song MJ, Lee KS, Lee YH, Ko SY, Lee YK. Congenital portosystemic shunts: prenatal manifestations with postnatal confirmation and followup. J Ultrasound Med. 2013 Jan;32(1):45-52. PubMed PMID: 23269709.

8. Knirsch W, Benz DC, Bühr P, et al. Catheter interventional treatment of congenital portosystemic venous shunts in childhood. Catheter Cardiovasc Interv 2016; 87: 1281-1292. 